\title{
Nanoengineered astronomical optics
}

\author{
E. F. Borra ${ }^{1,2}$, A. M. Ritcey ${ }^{1,3}$, R. Bergamasco ${ }^{1,3}$, P. Laird ${ }^{1,2}$, J. Gingras ${ }^{1,3}$, \\ M. Dallaire ${ }^{1,2}$, L. Da Silva ${ }^{1,2}$, and H. Yockell-Lelievre ${ }^{1,3}$ \\ ${ }^{1}$ Centre d'Optique, Photonique et Lasers, Université Laval, Québec, Qc, G1K 7P4, Canada \\ 2 Département de physique, de génie physique et d'optique, Université Laval, Québec, Qc, G1K 7P4, Canada \\ 3 Département de Chimie, Université Laval, Québec, Qc, G1K 7P4, Canada
}

Received 16 May 2003 / Accepted 12 January 2004

\begin{abstract}
We describe a technology for the fabrication of inexpensive and versatile mirrors through the use of a new type of nanoengineered optical material composed by the spreading of a self-assembling reflective colloidal film spread at the surface of a liquid. These new reflecting liquids offer interesting possibilities for astronomical instrumentation. For example, they can replace mercury in conventional rotating liquid mirrors. The main advantages offered include extremely low cost and, by coating a viscous liquid, the possibility of tilting the mirror by a few tens of degrees. We also have coated ferromagnetic liquids with these reflecting films. The resulting surfaces can be shaped by the application of a magnetic field, yielding reflecting surfaces that can have complicated shapes that can rapidly shift with time. These inexpensive and versatile optical elements could have numerous scientific and technological applications. Among possible astronomical applications, they could be used to make large inexpensive adaptive mirrors exhibiting strokes ranging from nanometers to several millimeters.
\end{abstract}

Key words. telescopes - instrumentation: miscellaneous - instrumentation: adaptive optics

\section{Introduction}

Most astronomical optical instruments use glass optics for the primary mirror of the telescope as well as for auxiliary optical elements. Polished glass surfaces are expensive and it is difficult, if not impossible, to give them complex shapes. It is therefore worthwhile to find new technologies that would enable us to build inexpensive optics, of all sizes, capable of having highly aspheric active surfaces.

Liquid mirrors offer such a solution. They take advantage of the fact that the surface of a liquid is very smooth and takes the shape of an equipotential surface so that one readily gets an optical quality surface. For example, the fact that the surface of a liquid rotating in a gravitational field takes the shape of a parabola has been used to make inexpensive mirrors having excellent surface qualities. The technology is young but its performance is well documented by laboratory tests (Borra et al. 1992; Girard \& Borra 1997; Tremblay \& Borra 2000) as well as by observations (Sica et al. 1995; Hickson \& Mulrooney 1998; Cabanac et al. 1998). One also can make liquid mirrors by shaping the equipotential surfaces with force fields other than gravity. Magnetic fields, for example, can be used with ferromagnetic liquids.

While mercury is useful for conventional rotating liquid mirrors because of its reasonably good reflectivity and low melting temperature, its density is high so that the container

Send offprint requests to: E. F. Borra, e-mail: borra@phy.ulaval.ca and bearing have to be sturdy and relatively costly. Pure mercury is slightly diamagnetic and cannot be easily deformed in a magnetic field. It can be rendered ferromagnetic, however it is difficult to obtain a stable mercury based ferromagnetic liquid (Charles \& Popplewell 1980). Furthermore, the high density of mercury necessitates large deforming forces and strong magnetic fields that require strong currents for the driving electromagnets, causing undue ohmic heating. It would therefore be useful to make magnetically shaped reflective liquids having better physical and chemical characteristics. We describe below such liquids based on colloidal surface films spread on liquid substrates.

\section{Deposition of reflective films on oil-based substrates}

The mirrors reported in this paper are comprised of a thin reflective layer of silver nanoparticles supported on a liquid substrate. Stable interfacial assemblies of silver nano-particles have been previously reported in the literature and are known as Metal Liquid-Like Films, or MELLFs (Yogev \& Efrima 1988; Gordon et al. 1989). MELLFs were originally used in chemical experiments to study the chemicals that coated the colloidal grains. We were intrigued by these colloidal systems that combine the optical properties of metals (reflectivity) with the fluidity of a liquid suspension and therefore seem well adapted to applications in the field of liquid optics. In an early paper (Borra et al. 1999, hereafter referred to as Paper I) we reported 


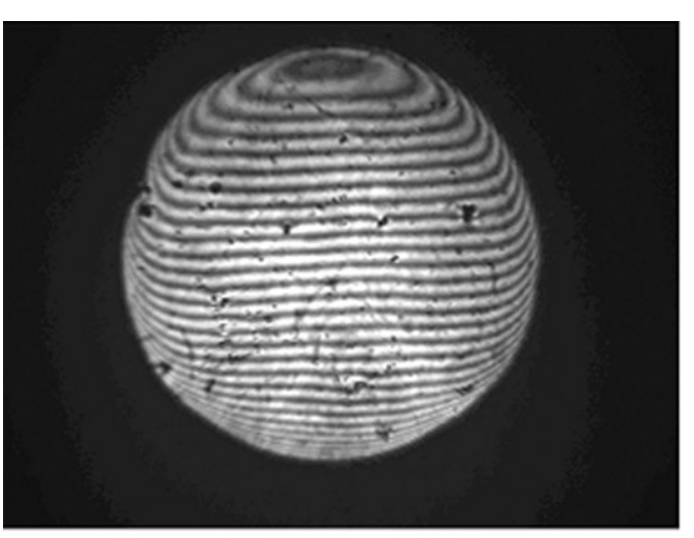

a)

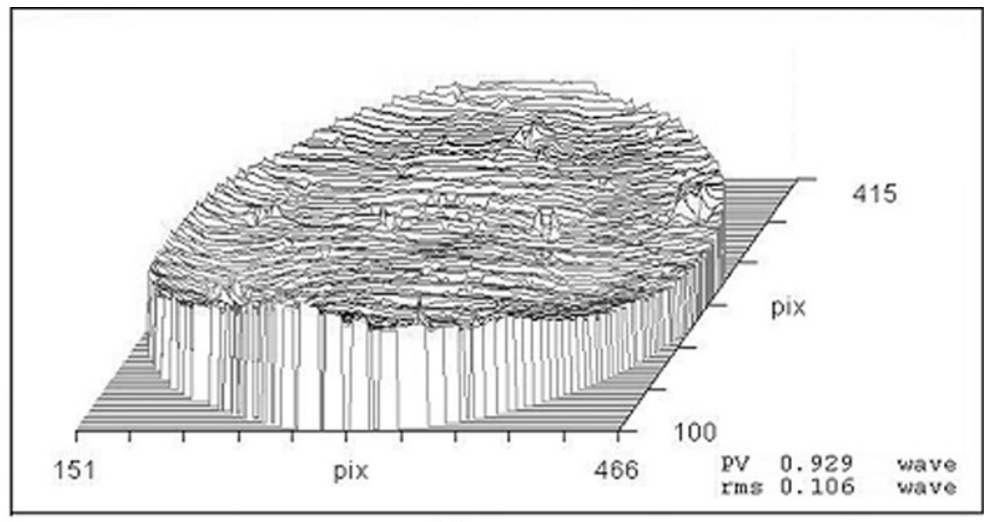

b)

Fig. 1. Interferogram obtained by measuring a $6.5 \mathrm{~cm}$ diameter sample of an oil surface coated with a MELLF. The resolution on the surface of the liquid was $0.1 \mathrm{~cm}$. Figure $1 \mathrm{~b}$ shows the wavefront obtained from that interferogram. They show an excellent optical quality, as evidenced by the 0.1 waves rms on the wavefront $(\lambda / 20$ on the surface).

on early experiments. The preparation of a MELLF involves the fabrication of silver nanoparticles coated with an organic protective layer. Experimental details have been previously described (Yockell-Lelievre et al. 2003, hereafter referred to as Paper II). In the present paper we report the successful subsequent spreading of the MELLF at the surface of an oil as described in the following procedure: an aqueous solution of an appropriate surfactant is first introduced into a container of desired dimensions. For a rotating mirror, the motorised rotation of the container is initiated before the MELLF, mixed with paraffin oil (50\% vol.), is slowly introduced onto the water surface. Phase separation spontaneously occurs and a reflective film of silver nanoparticles forms at the oil surface. At first optical quality is poor as the wet MELLF spreads irregularly at the surface of the oil. One must wait for the MELLF to spread uniformly over the surface and then dry. It typically takes about $45 \mathrm{~min}$ for the surface quality to improve sufficiently that optical measurements can be carried out.

\section{Nanoengineered liquid mirrors}

In Paper I we introduced nanoengineered mirrors that trapped colloidal particles at the interface between two liquids. In Paper II we discuss results obtained with water-based liquid mirrors where the colloidal particles are located at the top surface of water, a major improvement over Paper I where they were located at the interface between two liquids. In this section, we present results obtained with novel reflecting liquids made of silver coated oils. The ability to coat oil constitutes a major improvement of the technology, for the range of physical and chemical characteristics of the available liquids substrates is greatly extended. Furthermore, coating oil solves the evaporation problem which occurs with water-based liquids.

\subsection{Interferometry}

To develop the basic technology, we have mostly worked with flat mirrors since they are easier to handle and test interferometrically. Our tests are carried out with a Fizeau interferometer at a wavelength of $632.8 \mathrm{~nm}$.

Figure 1a shows an interferogram obtained by measuring a $6.5 \mathrm{~cm}$ diameter sample of one of our dried MELLF surfaces spread on vegetable oil while Fig. 1b shows the corresponding wavefront obtained from the interferogram. Both images indicate excellent optical quality. The resolution on the surface of the liquid was $0.1 \mathrm{~cm}$. Deviations from a flat surface are mostly caused by defects in a flat glass folding mirror used to observe the liquid rather than defects in the sample mirror. There is also some contribution from waves on the liquid surface that are induced by laboratory vibrations and from deformations at the edges due to surface tensions near the rim of the relatively small container. The waves could be dampened by using thinner liquid layers (Borra et al. 1992) or more viscous liquid substrates. Even with these preventable defects, we can see that the quality is excellent ( $\mathrm{rms}=0.106$ waves). Some minor problems with the coating technology must be noted. For example, large surfaces can be marred by a few bubbles, as well as dull spots caused by evaporating water droplets that leave solid esidues. Less than one percent of the surface is affected. We are presently developing techniques to minimize these defects.

\subsection{Reflectance curves}

We measured the reflectivity curves of our samples as a function of wavelength with a spectrophotometer. The reflectivity curves for two water-based, an oil-based mirror, as well as a coated ferrofluid are shown in Fig. 2. The better reflectivity of the water samples presumably are due to the fact that, at the time of this writing, we have spent far more time working with water and have been able to find improved experimental conditions to maximize the reflectivity; while the oil coating technique was only discovered recently. The difference between the two water-based curves illustrates some of the progress achieved in a few months work following Paper II. Similar optimization of the various experimental parameters affecting the reflectivity (such as the choice of surfactant and particle coating agent) has not yet been carried out on the oil based systems. 


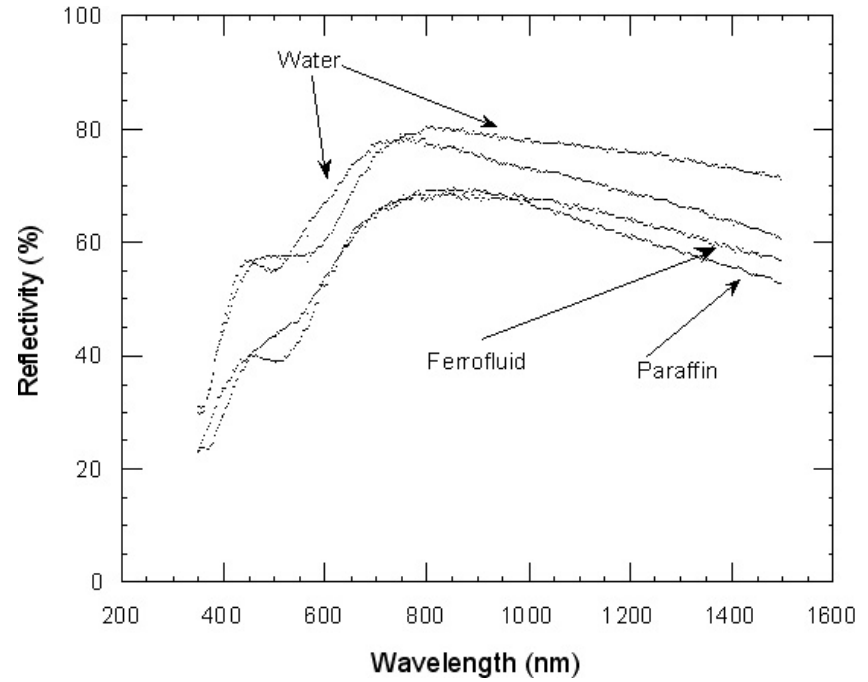

Fig. 2. Reflectivity curves for two water-based and an oil-based mirror, as well as a coated ferrofluid. The difference between the two water-based curves illustrates some of the progress achieved in a few months work following Paper II. The better reflectivity curve of the water based mirror is probably due to the fact that we have spent far more time working with water-based liquids.

We are confident that we can improve significantly our reflectivity curves with additional work currently in progress.

We have made a $1-\mathrm{m}$ diameter $\mathrm{f} / 2.5$ rotating mirror that uses a MELLF on liquid oil. We know from interferometry previously carried out on mercury and gallium liquid mirrors that liquid rotating surfaces are parabolical to a high accuracy so that we should expect that defects caused by the MELLF should have mostly high spatial frequencies. The knife-edge test, although not a very sophisticated one, is well-suited for our purpose since it can detect defects having very small amplitudes (as little as 1/1000th of a wave in some cases) for defects having high spatial frequencies. A knife-edge test image of the 1-m mirror is shown in Fig. 3. Comparing this image to previous knife-edge tests of liquid mirrors (Borra et al. 1985), we note the typical signature of a parabola, the absence of concentric ripples and the presence of small localized defects. The absence of ripples is due to damping in the more viscous liquid. The surface roughness on the mirror is consistent with the surface roughness shown in the interferogram of Fig. $1(\lambda / 20)$.

We have characterized the stability of these mirrors by measuring the variation of the reflectivity at $632.8 \mathrm{~nm}$ with time over a period of a few weeks. We find that the reflectivity decreases by about $8 \%$ over the first 10 days after a freshly made MELLF is deposited on oil surface. After that, the reflectivity stays constant for the next few weeks. We furthermore measured the variations of the full reflectivity curves over a one week period and found that the reflectivity decrease measured at $632.8 \mathrm{~nm}$ in fact only occurs at wavelengths shorter than of about $800 \mathrm{~nm}$. No significant variation is observed with time for longer wavelengths. Note that presumably, we can slow down the degradation of reflectivity with a proper choice of coating agent. Indeed, more recent, work done after this paper was first submitted, indicates that this is the case.
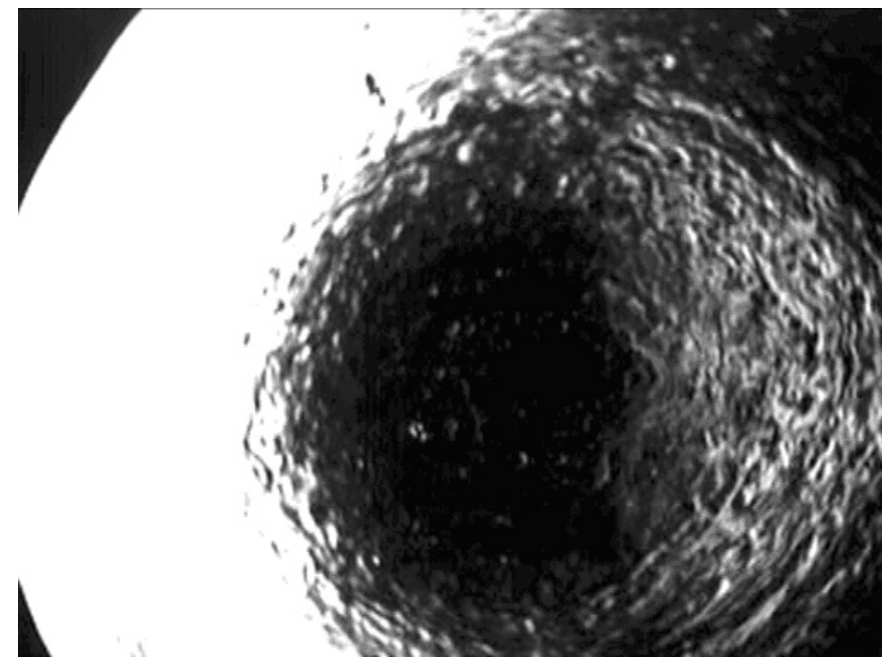

Fig. 3. Image obtained from a knife-edge test carried out on a 1-m diameter $\mathrm{f} / 2.5$ rotating parabolic liquid mirror composed of a MELLF spread on paraffin oil. We can clearly see the signature of a parabola. The surface roughness on the figure is consistent with the surface roughness measured for the wavefront of Fig. $1(\lambda / 20)$.

\section{Nanoengineered ferromagnetic liquid mirrors}

Ferrofluids are multi-phase liquids in which ferromagnetic particles are held as a colloidal suspension in a carrier liquid. This is a mature technology that is well-described in (Rosensweig 1992). A wide range of carrier liquids is available, the choice of carrier having a very strong impact on the hydrodynamic properties of the resulting ferrofluid. As a first approximation, the deformation of an inviscid magnetic liquid in a static magnetic field normal to the surface of the liquid is given by

$\delta h=B^{2}\left(\mu_{r}-1\right) /\left(2 \mu_{0} \rho g\right)$

where $\rho$ is the density of the ferrofluid and $B$ is the external magnetic field just above the ferrofluid surface. When the component of the magnetic field normal to the surface exceeds a critical value related to the interfacial tension and the density of the ferrofluid, a non-linear surface phenomenon instability, the Rosensweig instability, develops which renders the surface totally useless for our purpose. For typical light oil based ferrofluids the instability occurs around 84 Gauss, giving a maximum surface deformation for normal magnetic fields of the order of $1 \mathrm{~mm}$, well above the stroke requirement for adaptive optics. Other than that imposed by the Rosensweig instability, there is no fundamental limit to the height of a column of ferrofluid in a magnetic field, provided there is a sufficient supply of liquid in the container. Indeed Rosensweig (1992) has demonstrated a column of ferrofluid held in the magnetic field surrounding a current carrying vertical rod several $\mathrm{cm}$ high.

We have built a ferrofluidic mirror having a diameter of $80 \mathrm{~mm}$ with an actuator spacing of $5.5 \mathrm{~mm}$. The actuators consist of $5 \mathrm{~mm}$ diameter coils with soft ferrite cores that reduce current requirements by at least two orders of magnitude. Commands are generated using a desktop computer. The mirror surface was characterized with a Fizeau interferometer at a wavelength of $632.8 \mathrm{~nm}$. Deformations of up to $10 \mu \mathrm{m}$ have 


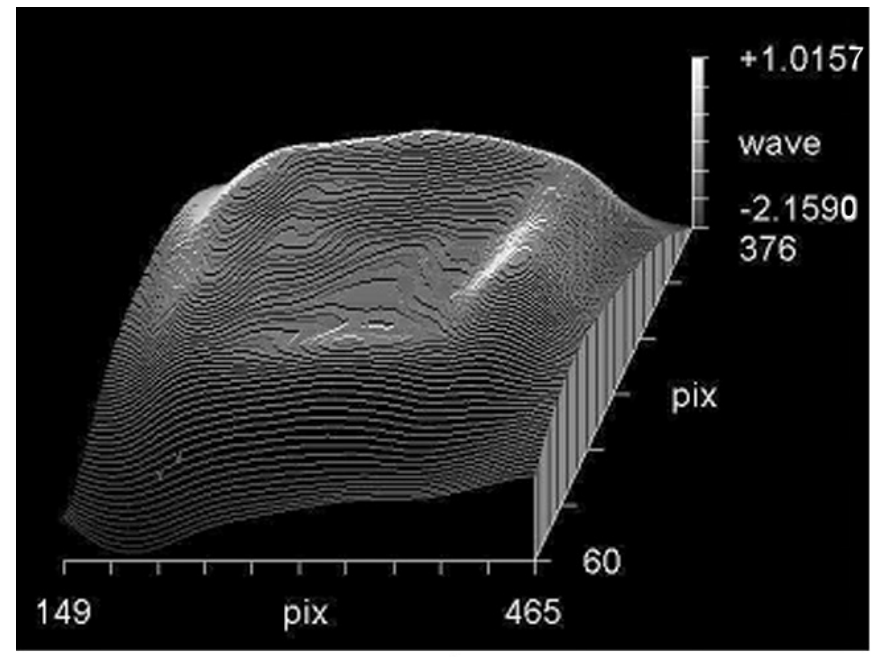

a)

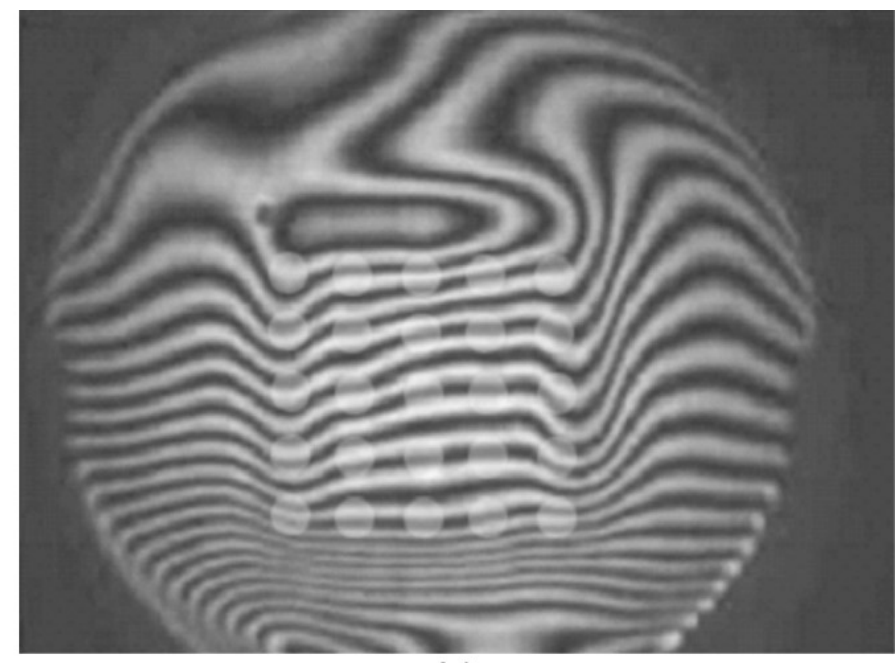

b)

Fig. 4. Piston term wavefront produced using the 25 central actuators in the ferrofluid mirror as well as the interferogram of the entire mirror. This wavefront was obtained on an uncoated ferrofluid. The array of small light grey disks show the positions of the actuators.

been measured; beyond which point the measurement becomes difficult due to the close fringe spacing. The magnetic fields needed to produce these deformations are small enough that no hysteresis is observed in the core material. This was confirmed with interferometer measurements of the surface up to $5 \mu \mathrm{m}$ as well as for deformations up to approximately $1 \mathrm{~mm}$ by measuring the position of a laser reflected from the surface during several actuator cycles to full deflection Qualitative measurements were also made of deformations on the order of several $\mathrm{mm}$ that were visible to the naked eye confirming the extremely large dynamic range of this type of mirror. The deformed surfaces appeared smooth to the unaided eye. The Rosensweig instability did not occur because the normal component of the magnetic field was below the critical value for the fluid used in these experiments.

As surface deformation depends on $B^{2}$, the actuators are unipolar and the mirror must be biased to $50 \%$ stroke before applying surface figures. Figure 4 shows an interferogram and the resulting wavefront produced using the 25 central actuators in the mirror. The array of light gray disks shows the actuator positions. The central plateau has an rms wavefront deviation from flatness of 0.06 waves. We are now upgrading our system to 100 actuators and developing algorithms to generate Zernike polynomials. Improved surface quality should also be possible but will require improvements to our control software.

Preliminary experiments show that the surface of a ferrofluid having a kinematic viscosity of $5 \mathrm{cps}$ is well behaved in response to a $20 \mathrm{~Hz}$ driving signal. Specifically, we have measured a $5 \mu \mathrm{m}$ stroke driven by a step function. Beyond $20 \mathrm{~Hz}$, experimental limitations made it difficult to determine the exact behaviour of the surface although indications are that the surface was not returning to zero before the next pulse, that is to say the fluid was not flowing fast enough to support actuation substantially faster than $20 \mathrm{~Hz}$. The scaling law frequency $\approx \sqrt{\text { amplitude }}$ predicts $40 \mathrm{~Hz}$ for $1 \mu \mathrm{m}$ deformation. A lower viscosity fluid should also give higher frequencies. It must be noted that the $20 \mathrm{~Hz}$ frequency results from a worse case scenario since gravity and surface tensions give the only restoring forces. In a working mirror, upward-pushing actuators can deform the surface at a speed that can be quite rapid, depending on the local magnetic field strength. Consequently, the hydrodynamic flow resulting from the upward-driving actuators will drive down the surface above the down-driving actuators at a speed faster than the one set by gravity and surface tensions. We therefore expect to be able to reach significantly higher frequencies. We intend to carry out more detailed experiments in the future. One of these experiments will address the question of surface wave propagation. Preliminary results suggest that any waves generated by the deflection of an actuator are damped much faster than $20 \mathrm{~Hz}$ for the fluid in question. We are confident that we can exceed $100 \mathrm{~Hz}$ for the mirror as a whole.

We have a simple theoretical model for surface deformations, which is still being improved, that neglects viscous drag, which reduces performance, and surface tensions which provides a restoring force thus improving actuator performance. The model predicts a maximum mirror speed of $700 \mathrm{~Hz}$ at $5 \mu \mathrm{m}$ dropping to $500 \mathrm{~Hz}$ at $10 \mu \mathrm{m}$. This is an order of magnitude higher than the observed behavior suggesting that the model is overly optimistic and that the deformable mirror as tested is not optimized for high speed. The behaviour of the mirror is currently being studied using a more complete model that incorporates surface tension and viscous effects using finite element methods.

Our work to date has suggested several improvements to the design of the driving electronics that are expected to improve the speed of the mirror. Additional measurements are planned with improvements in the measuring technique that will allow precise measurement of larger strokes with faster drive signals. Moreover, by studying different fluids it will be possible to better understand the influence of carrier fluid hydrodynamic properties on mirror response.

The influence function of a liquid deformable mirror, which is the deformation measured at an actuator due to an applied 
current in an adjacent coil, has a coupling in the range of $18-33 \%$ which is consistent with conventional deformable mirrors. Individual coils produce a Gaussian influence function however the vector nature of the magnetic fields generated by the coils produces small dips between actuators and some edge effects at the outside actuator positions. Proper selection of ferrofluid depth and coating film smoothes out these dips and the edge effects are handled by the control system. More work remains to optimize actuator geometry and ferrofluid thickness to further improve surface quality.

Power dissipation in the actuators of a deformable mirror is a common problem because astronomical optics must preserve seeing quality. The use of high magnetic susceptibility ferrofluids with low density allows operation at low coil currents. A $10 \mu \mathrm{m}$ deformation of a $2 \mathrm{~mm}$ thick ferrofluid requires a power dissipation of approximately $8 \mathrm{~mW}$ per coil. The stated power requirement is for the quasi-static case in which $\mathrm{I}^{2} \mathrm{R}$ heat dissipation in the coils and amplifier circuits dominate. One of the advantages of the ferromagnetic mirror over conventional solid DMs is the relatively trivial amount of work required to deform the surface. Even at $1 \mathrm{kHz}$, the work required to drive a liquid mirror to a $10 \mu \mathrm{m}$ stroke is calculated at less than $2 \times 10^{-8} \mathrm{~W}$, orders of magnitude less than the $\mathrm{I}^{2} \mathrm{R}$ losses. At this level, no active cooling was required at room temperature. Additionally, because the coils are low resistance and the currents required are quite small, no high voltage stage is required, in contrast with piezoelectric actuators.

\section{Discussion and conclusion}

This article reports on optical tests of mirrors made with a chemical technique that allows for the coating of the air-liquid interface of water or oil based substrates with a reflective layer of self-assembling metallic nanoparticles. This is a significant improvement over the work in Paper I, where the reflecting layer was trapped at the interface between two liquids. In the new configuration, the reflecting layer coats the first surface seen by incident light. The ability to coating oil, as opposed to water, offers significant advantages since it increases the number of available liquids and solves the evaporation problem of water-based liquids.

Interferometry shows that the coated surfaces have excellent optical qualities. Reflectivity measurements show peak values of the order of $80 \%$, for water based MELLFs which is less than the reflectivity of silver or aluminum coated glass but usable nevertheless. The reflectivity of oil-based MELLF is lower but this is presumably due to the fact that, being newer, we have not done as much work with them. Consider that, in practice, glass-coated mirrors are seldom recoated, hence degrade with time as they get covered with dust and dirt. This is especially a problem for telescope mirrors used in astronomy, the space and atmospheric sciences which are exposed to the elements. Our reflecting layers are inexpensive and easy to generate so that they can be refreshed often. We are confident that the reflectivities can be improved with additional work currently in progress. Knife-edge tests of a 0.5 -m diameter rotating mirror indicates a reasonable surface quality. We are currently working to improve the deposition technique to further improve surface quality and reflectivity.

We expect that nanoengineered liquid mirrors should be useful for scientific and engineering applications. The technology is interesting for large optics, such as large parabolic mirrors, because of its extremely low cost. Because the mirror basically is made of a 1-mm thick layer of liquid having the density of oil, a 10-m diameter coated liquid mirror would weigh less than $100 \mathrm{~kg}$. The costs of the mirror and supporting hardware, which depend on the weight of the reflecting liquid, should be quite low, probably of the order of a few tens of thousands of dollars. The quantities of chemicals needed are very small and the coating process itself is not labor demanding. This would usher in an era of inexpensive large telescopes readily available to astronomers. Making a 4-m class liquid mirror necessitates few resources and can easily be made in a University environment with mostly student labor. We have done it. Note also that, while conventional mercury mirrors can only be tilted by a few arcseconds, thus limiting their usefulness, rotating mirrors that use viscous liquids could be tilted by perhaps as much as a few tens of degrees (Paper I). Because they use viscous liquids, these mirrors would be quite insensitive to wind and vibrations. It is possible to make rotating parabolic mirrors with very small f/numbers. We have made a 1.4 -m diameter f/0.7 liquid mirror in our laboratory but considerably faster mirrors are certainly possible, something that would be extremely difficult to do with glass. By using containers having parabolic surfaces, it is still possible to have thin layers of liquids, which is necessary to dampen vibrations and wind-induced waves (Tremblay $\&$ Borra 2000). We parabolize the top surfaces of our containers by pouring a liquid polyurethane resin on the top rough surface of composite containers. The resin hardens after a few hours to give a solid surface that follows the desired parabola to a fraction of a millimeter (Tremblay \& Borra 2000).

Coated ferromagnetic liquids are interesting because they can be used to make versatile low-cost adaptive and active mirrors. Because their surfaces can be shaped with magnetic fields, one can generate complex, time varying surfaces that are difficult and expensive to make with conventional techniques. We have demonstrated a magnetically deformable ferromagnetic liquid mirror capable of producing strokes of more than $10 \mu \mathrm{m}$ with relatively low power requirements. The speed of this device for deformations up to $10 \mu \mathrm{m}$ is expected to exceed $100 \mathrm{~Hz}$. Further testing is planned to confirm this. These mirrors promise several advantages over adaptive mirrors presently available. Deformations of the order of a few millimeters are possible using this system although the power requirements and heat loads become significant. There should be essentially no limit in size so that active mirrors having diameters of the order of one meter having hundreds of thousands of actuators should be feasible. We estimate that the cost of the hardware should run at about 5 dollars/actuator so that mirrors with hundreds of thousands of actuators would cost of the order of a million dollars. Mass production of the actuators could presumably result in even lower costs. Meter-sized active mirrors with hundreds of thousands of actuators are needed for the new generation of extremely large telescopes. Extremely complex 
shapes would be achievable, the limit being set by the number of actuators and their diameters.

Preliminary experiments indicate that ferromagnetic liquid mirrors can be used in a tilted or even inverted configuration with the addition of a suitable biasing magnetic field. We have fabricated down-facing ferromagnetic liquid mirrors that appeared smooth to the unaided eye, but have not yet carried out optical testing. It may thus be possible to use a liquid mirror as an adaptive secondary located above the main mirror or in other interesting geometries. Because the surface of liquids can be deformed by a variety of other physical effects (e.g. electrical or thermal fields), nanoengineered optics constitutes a new class of versatile and inexpensive optical elements that could be used in a variety of astronomical instrumentation. We are beginning to investigate this technology.

This is a very young technology and there is a significant work to be done before it reaches the performance and robustness expected for routine scientific or industrial applications. The work carried out so far has been relatively simple and we have reached with ease several milestones with the relatively modest resources typically available in a Canadian University, boding well for the future.

Acknowledgements. This research was supported by the Natural Sciences and Engineering Research Council and The Canadian
Institute for Photonics Innovation. L.Vieira da Silva Jr. wishes to thank the Conselho Nacional de Desenvolvimento Científico e Tecnológico, $\mathrm{CNPq}$, Brazil for their financial support.

\section{References}

Borra, E. F., Beauchemin, M., Arsenault, R., \& Lalande, R. 1985, PASP, 97, 454

Borra, E. F., Content, R., Girard, L., Szapiel, S., Tremblay, L. M., \& Boily, E. 1992, ApJ, 393, 829

Borra, E. F., Ritcey, A., \& Artigau, E. 1999, ApJ, 516, L115 (Paper I)

Cabanac, R., Borra, E. F., \& Beauchemin, M. 1998, ApJ, 509, 309

Charle, S., \& Popplewell, J. 1980, Ferromagnetic liquids, 2 (NorthHolland Publishing Company)

Girard, L., \& Borra, E. F. 1997, Appl. Opt., 36, 25, 6278

Gordon, K. C., McGarvey, J. J., \& Taylor, K. P. 1989, J. Phys. Chem., 93, 6814

Hickson, P., \& Mulrooney, M. 1998, ApJS, 115, 35

Rosensweig, R. E. 1992, Magnetic Fluids, Scientific American, 136

Sica, R. J., Sargoytchev, S., Borra, E. F., Girard Argall, S., Sarrow, C. T., \& Flatt, S. 1995, Appl. Opt., 34, 6925

Tremblay, G., \& Borra, E. F. 2000, Appl. Opt., 39, 5651

Yogev, D., \& Efrima, S. 1988, J. Phys. Chem., 92, 5754

Yockell-Lelievre, H., Borra, E. F., Ritcey, A., \& Da Silva, L. 2003, Appl. Opt., 42, 1882 (Paper II) 